\title{
Hospederos alternativos de Magnaporthe grisea del trigo en Paraguay
}

\author{
Alternative hosts of Magnaporthe grisea of wheat in Paraguay
}

\author{
Alice Chávez $^{1 *}$ y Mohan Kohli ${ }^{2}$ \\ ${ }^{1}$ Cámara Paraguaya de Exportadores y Comercializadores de Cereales y Oleaginosas (CAPECO), Centro de \\ Investigación Hernando Bertoni, Instituto Paraguayo de Tecnología Agraria, Caacupé, Paraguay. \\ ${ }^{2}$ CAPECO, Av. Brasilia 840, Asunción, Paraguay. \\ * Autor para correspondencia (alicerocio@ hotmail.es) \\ Recibido: 09/06/2014; Aceptado: 18/04/2015. \\ http://dx.doi.org/10.18004/investig.agrar.2015.junio.54-59
}

\section{RESUMEN}

El brusone del trigo causado por Magnaporthe grisea (T.T. Hebert) M.E. Barr (anamorfo Pyricularia grisea Sacc.), tuvo su primera epidemia en Paraguay en 2002, causando pérdidas de más del $70 \%$ en campos sembrados en la primera quincena de abril. Este hongo presenta una amplia gama de hospederos entre los cuales se destacan el arroz y el trigo, así como otras gramíneas cultivadas, nativas y malezas. Hasta el momento no se conocen los hospederos alternativos de este hongo en Paraguay o la importancia del inóculo procedente de los mismos para el cultivo del trigo. El objetivo de este estudio es identificar los posibles hospederos alternativos de Magnaporthe grisea en el Centro de Investigación Capitán Miranda, Itapúa, y en el Campo Experimental Yhovy, Canindeyú, durante los ciclos 2013 y 2014. Para ello, muestras de malezas, con síntomas característicos del ataque del hongo, fueron recolectadas en ambas localidades. Estas muestras fueron puestas en cámara húmeda y sembradas sobre medio de cultivo PDA, e incubadas durante 5 días a $25^{\circ} \mathrm{C}$ en el laboratorio de Fitopatología del Centro de Investigación Hernando Bertoni, Caacupé. Posteriormente se realizó la identificación del hongo mediante observación al estereoscopio y microscopio. De las 21 especies recolectadas se constató la presencia del hongo en: Bromus catharticus, Brachiaria sp., Chloris gayana, Cyperus diffusus, Digitaria horizontalis y Lolium multiflorum. Así también se realizaron pruebas de patogenicidad con los aislados de Bromus catharticus, Digitaria horizontalis y Lolium multiflorum las cuales confirmaron la patogenicidad de dichos aislados sobre trigo.

Palabras clave: Magnaporthe grisea, hospederos alternativos, trigo.

\section{ABSTRACT}

The first epidemic of wheat blast disease caused by Magnaporthe grisea (T.T. Hebert) M.E. Barr (anamorfo Pyricularia grisea Sacc.), was reported in 2002, causing more than $70 \%$ of production losses in seeded fields in the first half of April in Paraguay. This fungus attacks a wide number of hosts, including wheat and rice, as well as large number of other grass species and local weeds. So far, there is no knowledge on alternative host species to wheat blast fungus or their contribution to inoculum load for wheat in Paraguay. Therefore, the objective of this study was to identify alternative host species of Magnaporthe grisea present in the Capitán Miranda Research Center, Itapúa, and in the Yhovy Experimental Station, Canindeyú, during 2013 and 2014 crop cycle. Weed samples of showing characteristic blast disease symptoms were collected from both locations. These samples were kept in the humid chamber and later the fungus was transferred to Potato Dextrose Agar, PDA, culture medium and incubated for five days at $25^{\circ} \mathrm{C}$ in the Phytopathology laboratory at Hernando Bertoni Research Center, Caacupé. Afterwards, the fungal identification was done by observation under stereoscope and microscope. From the 21 weed species collected in the field, the fungus was observed on Bromus catharticus, Chloris gayana, Cyperus diffusus, Digitaria horizontalis and Lolium multiflorum. Pathogenicity tests conducted with isolates collected from Bromus catharticus, Digitaria horizontalis and Lolium multiflorum confirmed their potential to infect wheat.

Key words: Magnaporthe grisea, wheat, alternative host species. 


\section{INTRODUCCIÓN}

El brusone del trigo es una enfermedad causada por Magnaporthe grisea (T.T. Hebert) M.E. Barr (anamorfo Pyricularia grisea Sacc.), que tuvo su primera epidemia en Paraguay en 2002, causando pérdidas de más del 70\% en siembras tempranas (Viedma y Morel 2002). Aun cuando la enfermedad es capaz de atacar a todas las partes de la planta, su infección más grave es observada en la espiga. El hongo ataca el raquis resultando en que la espiga no forme granos en la porción superior al punto de infección (Kohli et al. 2011).

Las condiciones ambientales que favorecen una epidemia a nivel de campo no son muy claras. Viedma (2009 y 2010), menciona que siembras tempranas de la primera quincena de abril, tienen mayor riesgo de ataque si se producen condiciones de alta temperatura y humedad en el mes de junio. Sin embargo en años húmedos, con prevalencia del fenómeno El Niño, los ataques de Magnaporthe pueden ocurrir independientemente de la fecha de siembra (Kohli et al. 2011).

Este hongo presenta una amplia gama de hospederos entre los cuales se destacan el arroz y el trigo, así como numerosas gramíneas cultivadas, nativas y malezas (Reis et al. 2005). Lima (2004) señala que la cebada, milleto, maíz, triticale, centeno, acevén y gramíneas nativas son hospederos alternativos de $M$. grisea. Hasta el momento no se conoce acerca de los hospederos alternativos de este hongo en el Paraguay o sobre la importancia del inóculo procedente de los mismos para el cultivo del trigo.

El objetivo de este estudio es identificar los posibles hospederos alternativos y determinar la patogenicidad de Magnaporthe grisea en dos regiones trigueras (Sur y Norte), representadas por el Centro de Investigación Capitán Miranda, Itapúa, y en el Campo Experimental Yhovy, Canindeyú, durante los ciclos 2013 y 2014.

\section{MATERIALES Y MÉTODOS}

Las muestras de hojas e inflorescencias de malezas, que presentaban síntomas característicos del ataque de Magnaporthe grisea, fueron recolectadas de las zonas aledañas a las parcelas de trigo. Todas las malezas que presentaban manchas elípticas, con borde marrón rojizo u oscuro y centro más claro en las hojas (Figura 1) fueron tomadas para su análisis en el laboratorio (Reis et al. 2005).
La recolección se llevó a cabo en dos oportunidades durante el 2013, la primera en el mes de agosto, en el Centro de Investigación Capitán Miranda, Itapúa; y la segunda en el mes de diciembre tanto en Itapúa como en el Campo Experimental Yhovy, Canindeyú. Durante el 2014, las recolecciones se llevaron a cabo en el mes de julio en el Campo Experimental Yhovy; y en el mes de octubre en el Centro de Investigación Capitán Miranda. En total se recolectaron 19 especies de la familia Poaceae, una de la familia Cyperaceae y una de la familia Commelinaceae. La lista de las especies recolectadas se presenta en la tabla 1.

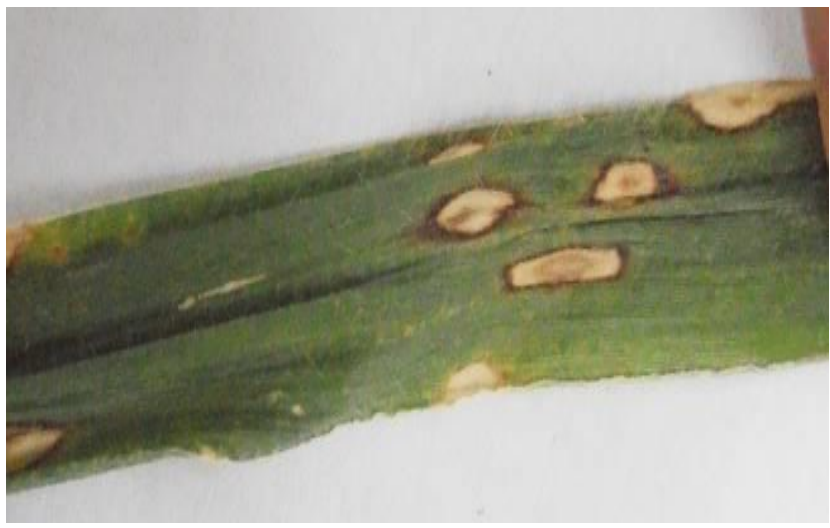

Figura 1. Síntomas característicos del ataque de Magnaporthe grisea en las gramíneas.

\section{Diagnóstico e identificación de Magnaporthe grisea}

Los estudios de diagnosis e identificación del hongo se realizaron en el Laboratorio de Fitopatología del Centro de Investigación Hernando Bertoni, Caacupé. Para ello, pequeñas porciones de la parte afectada fueron desinfectados mediante inmersión en solución de alcohol al $70 \%$ durante 30 segundos y después en hipoclorito de sodio (55 $\mathrm{g} \mathrm{Cl} \mathrm{L}^{-1}$ agua), por un minuto. Posteriormente fueron enjuagados tres veces con agua destilada esterilizada y secados sobre papel absorbente. Estas porciones fueron sembradas en placas de Petri con medio de cultivo PDA (Papa, Dextrosa, Agar), e incubados a $25^{\circ} \mathrm{C}$ durante cinco días.

Así también una parte de las muestras fue incubada en cámara húmeda a la misma temperatura y durante el mismo tiempo. Transcurrido el periodo de incubación, se realizó la identificación mediante observación al estereoscopio y microscopio, utilizando la clave de Barnett y Hunter (1998). 
Tabla 1. Lista de especies de malezas recolectadas en el Centro de Investigación Capitán Miranda (CICM), Itapúa y Campo Experimental Yhovy (CEY), Canindeyú durante el ciclo 2013.

\begin{tabular}{|c|c|c|c|c|c|c|}
\hline \multirow{2}{*}{\multicolumn{2}{|c|}{ Especies recolectadas }} & \multicolumn{5}{|c|}{ Lugar y época de recolección } \\
\hline & & \multicolumn{3}{|c|}{2013} & \multicolumn{2}{|c|}{2014} \\
\hline \multirow[t]{2}{*}{ Nombre común } & \multirow[t]{2}{*}{ Nombre científico } & \multicolumn{2}{|c|}{ CICM } & \multirow{2}{*}{$\begin{array}{c}\text { CEY } \\
\text { Dic }\end{array}$} & \multirow{2}{*}{$\begin{array}{c}\text { CICM } \\
\text { Oct }\end{array}$} & \multirow{2}{*}{$\begin{array}{c}\text { CEY } \\
\text { Jul }\end{array}$} \\
\hline & & Ago & Dic & & & \\
\hline Avena negra & Avena strigosa & $\checkmark$ & & & $\checkmark$ & \\
\hline Acevén & Lolium multiflorum & $\checkmark$ & & & $\checkmark$ & \\
\hline Aguara ruguai & Andropogon sp. & $\checkmark$ & & & & \\
\hline Brachiaria & Brachiaria sp. & $\checkmark$ & & & & $\checkmark$ \\
\hline Brachiaria & Brachiaria decumbens & $\checkmark$ & $\checkmark$ & & & \\
\hline Brachiaria & Brachiaria humidicola & & $\checkmark$ & & & \\
\hline Brachiaria & Brachiaria mutica & & $\checkmark$ & & & \\
\hline Bromus & Bromus catharticus & $\checkmark$ & $\checkmark$ & & $\checkmark$ & \\
\hline Cebadilla & Digitaria horizontalis & & $\checkmark$ & $\checkmark$ & $\checkmark$ & $\checkmark$ \\
\hline Cyperus & Cyperus diffusus & & $\checkmark$ & & & \\
\hline Kapi'i pororó & Digitaria insularis & $\checkmark$ & $\checkmark$ & $\checkmark$ & & $\checkmark$ \\
\hline Kapi ati & Cenchrus ciliaris & & $\checkmark$ & & & \\
\hline Maíz & Zea mays L. & & & & & $\checkmark$ \\
\hline Pasto colonial & Panicum maximum & $\checkmark$ & & & & $\checkmark$ \\
\hline Pasto estrella & Cynodon nlenfluensis & $\checkmark$ & $\checkmark$ & & & $\checkmark$ \\
\hline Pasto Rhodes & Cloris gayana & $\checkmark$ & $\checkmark$ & & & $\checkmark$ \\
\hline Pata de gallina & Eleusine indica & & $\checkmark$ & & & \\
\hline Santa Lucía & Commelina sp. & & & & & $\checkmark$ \\
\hline Sin nombre común & Imperata brasiliensis & & $\checkmark$ & & & \\
\hline Setaria & Setaria geniculata & & $\checkmark$ & $\checkmark$ & & $\checkmark$ \\
\hline Sorgo & Sorghum halepense & & $\checkmark$ & $\checkmark$ & & $\checkmark$ \\
\hline
\end{tabular}

\section{Pruebas de patogenicidad}

Hasta la fecha, las pruebas de patogenicidad se llevaron a cabo con los aislados de B. catharticus, D. horizontalis y L. multiflorum; sobre tres materiales de trigo: Canindé 1, Itapúa 70 y Canindé 11; utilizándose para cada aislado 3 macetas con dos plantas de cada material. La inoculación se llevó a cabo mediante aspersión empleando $50 \mathrm{~mL}$ de una suspensión de $210^{5}$ conidios $\mathrm{mL}^{-1}$ de cada aislado. Luego de la inoculación las plantas fueron mantenidas en una cámara con humedad por 24 horas para favorecer la infección. La evaluación se llevó a cabo una semana después de la inoculación y se caracterizaron las lesiones de acuerdo con la clasificación propuesta por Urashima et al. (2004) donde:

$0=$ Ausencia de infección

$1=$ Lesiones oscuras y diminutas denominadas

"cabeza de alfiler"

$2=$ Pequeñas lesiones mayores que las de tipo 1 , de color marrón a negro sin centro distinguible $3=$ Lesiones redondeadas con centro ceniza, denominadas "mancha ocular" 4= Lesiones típicas de susceptibilidad, elípticas y con centro ceniza.
Las lesiones de tipo 3 y 4 son consideradas reacciones de susceptibilidad, mientras que las de tipo 1 y 2 son consideradas de tipo resistente ya que no esporulan cuando son sometidas a condiciones favorables de temperatura y humedad.

\section{RESULTADOS Y DISCUSIÓN}

\section{Diagnóstico e identificación de Magnaporthe grisea}

Las observaciones realizadas con estereoscopio y microscopio (Figura 2) sobre las muestras colectadas, confirmaron la presencia de $M$. grisea en 6 de las 21 especies colectadas. La lista de las especies en cuyas muestras se confirmó su presencia se presenta en la tabla 2.

Sobre las muestras colectadas en Capitán Miranda durante el ciclo 2013 en el mes de agosto, confirmaron la presencia de $M$. grisea en las hojas de "bromus" (B. catharticus) y "pasto Rhodes" (C. gayana) así como en las hojas e inflorescencias de "acevén" (L. multiflorum). En las muestras recolectadas durante el mes de diciembre 
en Capitán Miranda, se constató la presencia del hongo en: C. diffusus, B. catharticus, y D. horizontalis (Figura $3)$.

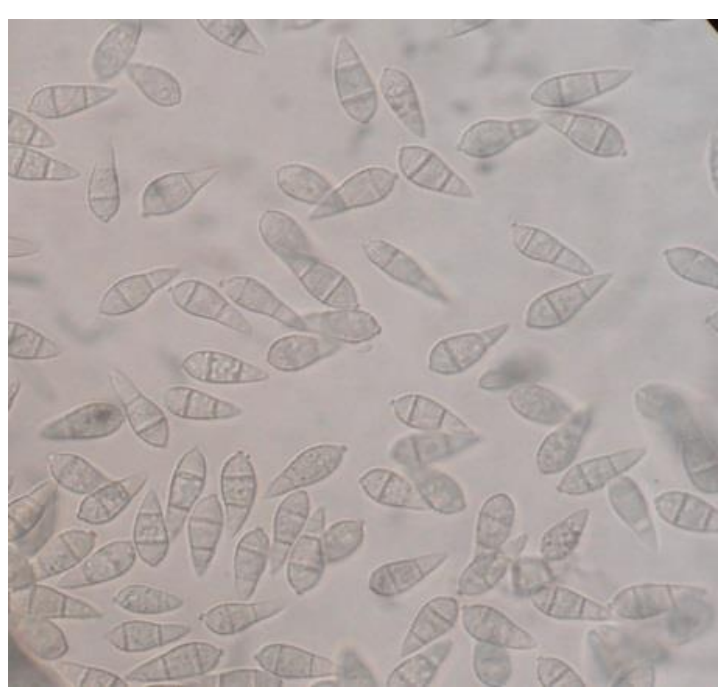

Figura 2. Conidios de Magnaporthe grisea presentes en muestras recolectadas en el Centro de Investigación Capitán Miranda (CICM), Itapúa y Campo Experimental Yhovy (CEY).
Tabla 2. Lista de especies con Magnaporthe grisea, recolectadas en el Centro de Investigación Capitán Miranda (CICM), Itapúa y Campo Experimental Yhovy (CEY), Canindeyú.

\begin{tabular}{ccccc}
\hline & 2013 & & \multicolumn{2}{c}{2014} \\
\hline \multicolumn{2}{c}{ CICM } & CEY & CICM & CEY \\
Agosto & Diciembre & Diciembre & Octubre & Julio \\
\hline B. & C. & D. & L. & D. \\
catharticus & diffusus & horizontalis & multiflorum & horizontalis \\
C. gayana & B. & & B. & Brachiaria \\
& catharticus & & catharticus & sp \\
L. & D. & & D. & \\
multiflorum & horizontalis & & horizontalis & \\
\hline \multicolumn{2}{c}{} & & &
\end{tabular}

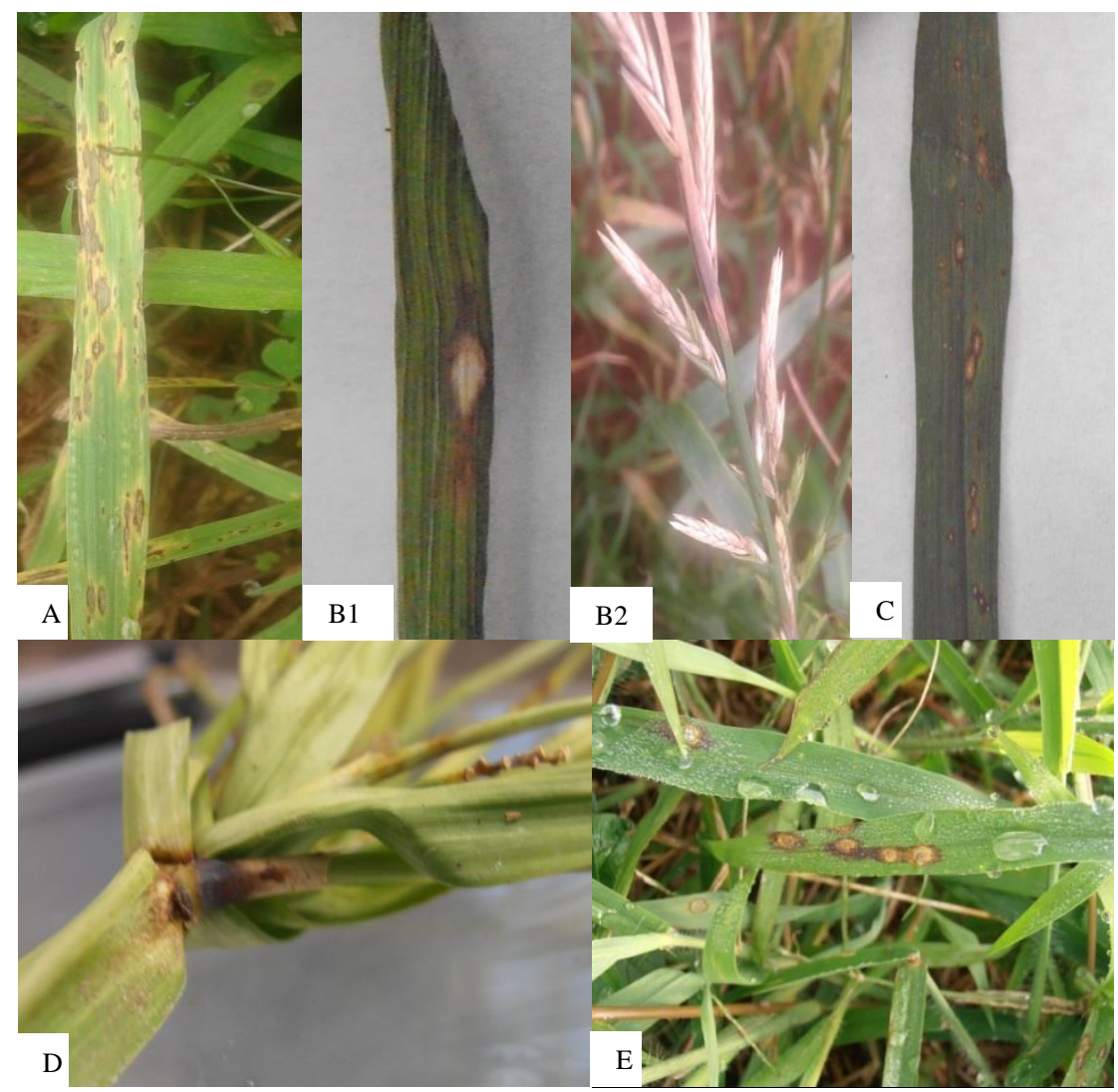

Figura 3. Síntomas observados en las muestras colectadas en el CICM durante los ciclos 2013 y 2014, (A) hojas de Bromus catharticus, (B1- B2) hoja e inflorescencia de Lolium multiflorum, (C) hoja d Chloris gayana, (D) inflorescencia de Cyperus diffusus y (E) hojas de Digitaria horizontalis. 
En cuanto a las muestras recolectadas en Yhovy, Canindeyú, durante el 2013 se observó la presencia de Magnaporthe solo en D. horizontalis. Durante el 2014, se observó la presencia del hongo en D. horizontalis y Brachiaria sp. Esto confirma lo mencionado por Gutiérrez et al. (2000) y Verzignassi et al. (2012) quienes reportaron el ataque de Magnaporthe en Brachiaria brizantha y Brachiaria extensa, respectivamente.

Así también Prabhu et al. (1992) y Urashima et al. (1993) reportan otras malezas adicionales de la familia Gramineae (Cencrhus echinatus, Eleusine indica, Digitaria sanguinalis, Brachiaria plantaginea, Echinocloa crusgalli, Pennisetum setosum, Hyparrhenia ruffa y Rhynchelytrum roseum) como posibles fuentes de inóculo del hongo. Recientemente, Perelló et al. (2014) mencionan haber aislado con éxito Magnaporthe oryzae en las siguientes especies Stenotaphrum secundatum, Setaria sp., Eleusine sp., Bromus unioloides, Lolium perenne, Echinochloa cruz-galli, Avena fatua y Cynodon dactylon. Muchas de estas malezas son comunes en los campos nacionales.

Por otra parte, no fue posible aislar el hongo de las muestras de avena negra (Avena strigosa) recolectadas en Itapúa, aunque Viedma (2010) menciona su identificación en parcelas de Colonias Unidas en el año 2005. Mehta et al. (2006), incorporaron la avena negra a la lista de fuentes probables de infección primaria y recientemente Marangoni et al. (2013) agregaron la avena blanca como especie susceptible al brusone de trigo.

De los estudios realizados en Japón, Ou (1985) menciona que Hashioka identificó $M$. grisea en especies cultivadas y espontáneas de las familias Cannaceae, Zingiberaceae, Musaceae, Gramineae, Cyperaceae y Commelinaceae.

Cabe destacar, además, que varias de las especies recolectadas en las que no se encontró Magnaporthe grisea no pueden ser descartadas como hospederos alternativos a nivel local, considerando los numerosos reportes que demuestran su importancia en la región.

\section{Pruebas de patogenicidad}

Las pruebas de patogenicidad realizadas arrojaron resultados positivos para los tres aislados empleados, el tipo de lesión observado en los diferentes materiales se presenta en la tabla 3 .
Tabla 3. Tipo de lesión de acuerdo con la escala de Urashima et al. (2004), causado por los aislados de Magnaporthe grisea en los diferentes materiales de trigo.

\begin{tabular}{cccc}
\hline Aislado & Itapúa 70 & Canindé 1 & Canindé 11 \\
\hline Bromus catharticus & 0 & 0 & 1 \\
Digitaria horizontalis & 3 & 4 & 3 \\
Lolium multiflorum & 2 & 0 & 3 \\
\hline
\end{tabular}

De los tres aislados empleados el que presentó mayor virulencia fue el aislado de $D$. horizontalis, ya que causó lesiones de tipo susceptible en los tres materiales, así también el aislado de L. multiflorum causó este tipo de lesión en el Canindé 11. En concordancia, Perelló et al. (2014), observaron síntomas similares a los de tipo 1 y 3 , al inocular aislados de trigo y otras gramíneas en los cultivares BioINTA 3004 y Baguette 18 con severidades entre 2,7 y $64,3 \%$.

Estos resultados dan una idea de la variabilidad patogénica existente y solo un estudio exhaustivo de distintas especies alternativas presentes en el país proporcionará el cabal entendimiento de su importancia y papel que estas juegan en la epidemiología del brusone en el trigo. Además, esos estudios podrían contribuir con el mejoramiento de la base genética de la resistencia en trigo y desarrollar una estrategia integral de control que en el futuro pueda alcanzar un alto nivel de eficacia.

\section{CONCLUSIONES}

Estos estudios, los primeros realizados para identificar la presencia de Magnaporthe grisea en especies alternativas de malezas gramíneas confirman la infección por el hongo en Bromus catharticus, Brachiaria sp., Lolium multiflorum, Chloris gayana, Cyperus diffusus y Digitaria horizontalis.

Las pruebas de patogenicidad realizadas con los aislados de Bromus catharticus, Digitaria horizontalis y Lolium multiflorum sobre las plantas de trigo confirman el papel de estas especies como huéspedes alternativos para Magnaporthe grisea.

\section{AGRADECIMIENTOS}

El presente estudio fue realizado con el apoyo financiero del proyecto Pyricularia en trigo USDA y el Programa de Investigación de Trigo del Instituto Paraguayo de 
Tecnología Agraria (IPTA) a través del convenio IPTA/CAPECO/INBIO.

\section{REFERENCIAS BIBLIOGRÁFICAS}

Barnett, HL; Hunter, BB. 1998. Ilustrated genera imperfect fungi. 4 ed. The American Phytopathological society. Minnesota, US. 218 p.

Gutiérrez, S; Mazzanti de Castañon, M; Galmarini, M. 2000. Avances en el conocimiento de hospederos espontáneos de Magnaporthe grisea en Argentina. (en línea). Comunicaciones científicas y tecnológicas, Universidad Nacional del Nordeste. Consultado 06 ago. 2013. Disponible en: http://www.unne.edu.ar/unn evieja/Web/cyt/cyt/2000/5_agrarias/a_pdf/a_043.pdf

Kohli, MM; Mehta, YR; Guzman, E; Viedma, LQ; Cubilla, L. 2011. Pyricularia Blast- A threat to Wheat Cultivation. Czech J Genet Plant Breed 47: S130S134. 2011 (Speacial Issue).

Lima, MIPM. 2004. Giberela ou Brusone? Orientaçoes para a identificação correta dessas enfermidades em trigo e em cevada. (en línea). Documentos on line. Embrapa Trigo. Consultado 30 abr. 2013. Disponible en: http://www.cnpt.embrapa.br/biblio/do/p_do40.htm

Mehta, YR; Nunes, MP; Oliveira, JC. 2006. Occurrência de brusone em aveia no Estado do Paraná. In: Resultados Experimentais. XXVI Reunião da Comissão Brasileira de Pesquisa de Aveia, 4-6 de abril, FAPA, Guarapuava, Paraná, 55-57.

Marangoni, M; Nunes, M; Fonseca, N; Metha, Y. 2013. Pyricularia blast on White oats - a new threat to wheat cultivation. Tropical Plant Pathology 38(3):198-202.

Ou, SH. 1985. Rice diseases. 2 ed. Kew, Surrey. England. Commonwealth Mycological Institute. Consultado 31 mar. 2014. Disponible en: http://books.google.com.p $\mathrm{y} /$ books? $\mathrm{id}=\mathrm{k} 3 \mathrm{mewv} 9 \mathrm{nMoC} \&$ printsec $=$ frontcover $\& \mathrm{dq}$ $=\mathrm{Ou}+\mathrm{SH}+\mathrm{Rice}+$ diseases $\& \mathrm{hl}=\mathrm{es} \& \mathrm{sa}=\mathrm{X} \& \mathrm{ei}=\mathrm{qrY} 5 \mathrm{U} 4 \mathrm{z}$ 6OqLLsAS2q4AI\&redir_esc $=\mathrm{y} \# \mathrm{v}=$ onepage $\& \mathrm{q}=\mathrm{Ou} \%$ 20SH\%20Rice\%20diseases $\& \mathrm{f}=$ false
Perelló, A; Martínez, I; Molina, M. 2014. First report of virulence and effects of Magnaporthe oryzae isolates causing wheat blast in Argentina. Plant Disease. ISSN 0191-2917.

Prabhu, AS; Filippi, MC; Castro, N. 1992. Pathogenic variation among isolates of Magnaporthe grisea infecting rice, wheat, and grasses in Brazil. Tropical Pest Management 38: 367-371.

Reis, EM; Casa, RT; Forcelini, CA. 2005. Doenças do trigo (Triticum aestivum L.). In Kimati, H; Amorim, L; Rezende, J; Bergamin, A; Camargo, L. Manual de Fitopatologia: doenças das plantas cultivadas. 4 ed. São Paulo, BR. Ceres. v. 2, 663 p.

Urashima, AS; Igarashi, S; Kato, H. 1993. Host range, mating type, and fertility of Magnaporthe grisea from wheat in Brazil. Plant Disease 12: 11-16.

Urashima, AS; Lavorent, NA; Goulart, ACP; Metha, YR. 2004. Resistance spectra of wheat cultivars and virulence diversity of Magnaporthe grisea isolates in Brazil. Fitopatologia Brasileira 29: 511-518.

Verzignassi, JR; Poltronieri, LS; Benchimol, RL; Santos de França, SK; de Arruda, Eudes; Dornelas, C. 2012. Magnaporthe grisea: novo patógeno em Brachiaria brizantha cv. Marandú no Pará. Summa Phytopathologica 38 (3): 254.

Viedma, LQ; Morel, W. 2002. Añublo o Piricularia del Trigo. Díptico. MAG/DIA/CRIA. Programa de Investigación de Trigo, CRIA, Capitán Miranda, Itapúa.

Viedma, LQ. 2009. Manejo integrado de enfermedades en trigo. In: Kohli, M; Cubilla, LE; Viedma, L.Segundo seminario nacional de trigo "Del grano al Pan". Asunción, PY. CAPECO, INBIO. 140 p.

Viedma, LQ. 2010. Manejo integrado de mancha amarilla y la Piricularia en el cultivo de trigo en Paraguay. In: Kohli, M; Cubilla, LE; Cabrera, G. Tercer seminario nacional de trigo "Del grano al Pan". Asunción, PY. CAPECO, INBIO. 168 p. 